\title{
Enhanced transmission through a subwavelength aperture using metamaterials
}

Atilla Ozgur Cakmak, Koray Aydin, Evrim Colak, Zhaofeng Li, Filiberto Bilotti, Lucio Vegni, and Ekmel Ozbay

Citation: Appl. Phys. Lett. 95, 052103 (2009); doi: 10.1063/1.3195074

View online: https://doi.org/10.1063/1.3195074

View Table of Contents: http://aip.scitation.org/toc/apl/95/5

Published by the American Institute of Physics

\section{Articles you may be interested in}

Gratingless enhanced microwave transmission through a subwavelength aperture in a thick metal plate Applied Physics Letters 81, 4661 (2002); 10.1063/1.1527704

Sensing with toroidal metamaterial

Applied Physics Letters 110, 121108 (2017); 10.1063/1.4978672

Ultra-thin metasurface microwave flat lens for broadband applications

Applied Physics Letters 110, 224101 (2017); 10.1063/1.4984219

Ultra-broadband microwave metamaterial absorber

Applied Physics Letters 100, 103506 (2012); 10.1063/1.3692178

Split ring resonator sensors for infrared detection of single molecular monolayers

Applied Physics Letters 95, 043113 (2009); 10.1063/1.3194154

Subwavelength resolution with a negative-index metamaterial superlens

Applied Physics Letters 90, 254102 (2007); 10.1063/1.2750393
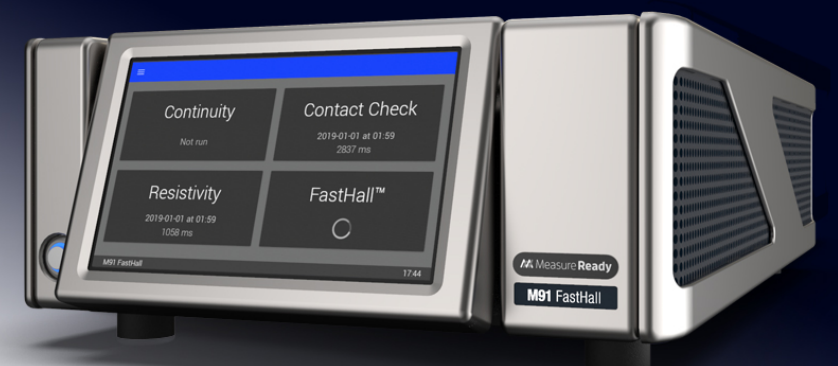

\section{Measure Ready M91 FastHall ${ }^{\text {TM }}$ Controller \\ A revolutionary new instrument for complete Hall analysis}




\title{
Enhanced transmission through a subwavelength aperture using metamaterials
}

\author{
Atilla Ozgur Cakmak, ${ }^{1, a)}$ Koray Aydin, ${ }^{1,2}$ Evrim Colak, ${ }^{1}$ Zhaofeng Li, ${ }^{1}$ Filiberto Bilotti, ${ }^{3}$ \\ Lucio Vegni, ${ }^{3}$ and Ekmel Ozbay ${ }^{1}$ \\ ${ }^{1}$ Department of Electrical and Electronics Engineering, Nanotechnology Research Center \\ and Department of Physics, Bilkent University, 06800 Ankara, Turkey \\ ${ }^{2}$ Thomas J. Watson Laboratories of Applied Physics, California Institute of Technology, Pasadena, \\ California 91125, USA \\ ${ }^{3}$ Department of Applied Electronics, University of Roma Tre, 00146 Rome, Italy
}

(Received 18 February 2009; accepted 15 July 2009; published online 4 August 2009)

\begin{abstract}
We report an enhanced transmission through a single circular subwavelength aperture that is incorporated with a split ring resonator (SRR) at the microwave regime. Transmission enhancement factors as high as 530 were observed in the experiments when the SRR was located in front of the aperture in order to efficiently couple the electric field component of the incident electromagnetic wave at SRR's electrical resonance frequency. The experimental results were supported by numerical analyses. The physical origin of the transmission enhancement phenomenon was discussed by examining the induced surface currents on the structures. (C) 2009 American Institute of Physics. [DOI: 10.1063/1.3195074]
\end{abstract}

The interest in transmission through subwavelength apertures has recently rapidly increased. ${ }^{1,2}$ The topic was addressed during the $1940 \mathrm{~s}$, where Bethe ${ }^{3}$ put forward his theoretical analysis suggesting relatively poor transmission figures at the output side of a subwavelength aperture. The problem remained a major challenge until the pioneering work of Ebbesen et al. ${ }^{4}$ From that moment on, the main focus has been shifted to elucidate the enhancement mechanism for subwavelength apertures. ${ }^{5}$ Researchers have set out to identify the role of the surface plasmons and to offer physical explanations for the transmission enhancement phenomenon. 6,7 Methods have been sought to effectively guide the incoming electromagnetic (EM) wave into the subwavelength aperture that will in return result in enhanced transmission. A slightly different approach was adapted in a paper by Alu et al., ${ }^{8}$ in which a matematerial cover over the aperture was theoretically shown to produce enhanced directional transmission by minimizing the diffraction losses. On the other hand, split ring resonators (SRRs) had already gained a well-established background in the metamaterial community. ${ }^{9}$ Innovative models were developed for SRRs that were placed inside a small circular metallic aperture. ${ }^{10}$ Subsequently, Aydin et al. ${ }^{11}$ proposed in a rather recent work an alternative method for transmission enhancement by putting a SRR in front of a subwavelength aperture.

We present here a more detailed analysis of the enhanced transmission process by focusing on an electrically coupled SRR that was made to cooperate with a subwavelength aperture. The resonator nature of the SRR was exploited to concentrate the fields in the vicinity of a subwavelength aperture to facilitate considerable improvement in the transmission factors at microwave frequencies. Besides, the dependence of the resonance frequency of the SRRs on the enhanced transmission was studied by employing three different SRRs in the simulations and experiments. Induced sur-

\footnotetext{
${ }^{\text {a) }}$ Author to whom correspondence should be addressed. Electronic mail: atilla@ee.bilkent.edu.tr.
}

face currents were calculated in order to discuss the enhancement mechanism. The transmission improvement figures were obtained as a function of various aperture radii.

Three distinct SRR designs with the labeled dimensions, which are shown in Fig. 1(a), were used. SRRs are deposited on a dielectric printed circuit board (PCB) with a thickness of $1.6 \mathrm{~mm}$ and a dielectric constant of $\varepsilon=3.85$. The deposited copper thickness is $30 \mu \mathrm{m}$. Samples A, B, and C have split widths of $w_{A}=0.2 \mathrm{~mm}, w_{B}=0.4 \mathrm{~mm}$, and $w_{C}=0.6 \mathrm{~mm}$. A closed ring resonator (CRR) was obtained by shortening the split of sample A. Each one of the CRR and SRRs possesses

(a)
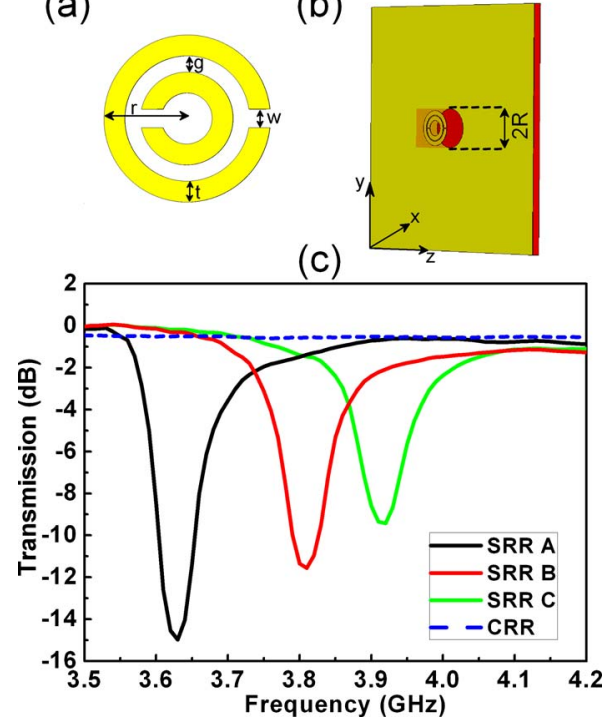

FIG. 1. (Color online) (a) The SRR configuration and the labeled SRR design dimensions: split width $(w)$, the distance between the inner and outer rings $(g=0.2 \mathrm{~mm})$, the metal width $(t=0.9 \mathrm{~mm})$, and the outer radius $(r=3.6 \mathrm{~mm})$. (b) The experimental setup: SRR is attached to the aperture with a diameter of $2 R$. SRR is shifted by an amount of $R$ (in the $-z$ direction) with respect to the aperture. (c) Measured transmission results for sample SRR A (solid black line), B (solid red line), C (solid green line), and the CRR (dashed blue line). 
(a)

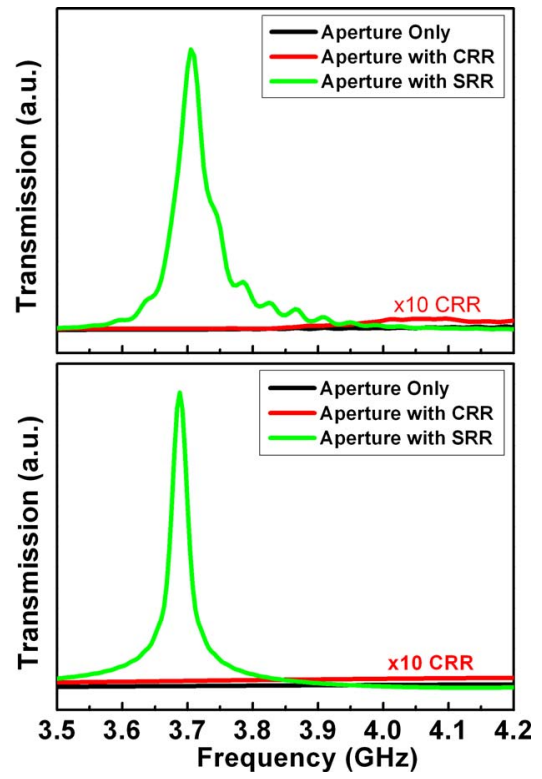

FIG. 2. (Color online) (a) Experimental and (b) simulation results of the transmission spectra: aperture only (solid black line), aperture with CRR (solid red line), and aperture with sample SRR A (solid green line).

a supporting PCB of $8 \times 8 \mathrm{~mm}^{2}$. An aperture with a radius of $R=4 \mathrm{~mm}$ was prepared by etching the copper on a single side copper-coated separate PCB [Fig. 1(b)]. The metal side of the resonators was affixed to the metal side of the PCB. There was a separation distance of approximately $0.1 \mathrm{~mm}$ between the two metal faces. Conventional waveguide antennas were connected to the Agilent N5230A network analyzer to collect the transmission results. The transmission characteristics of the SRR samples are depicted in Fig. 1(c). The incident beam had an electric field component along the $y$ axis [see Fig. 1(b) for directions]. The electrical resonance of the single SRRs was observed when the SRR plane was perpendicular to the propagation of the incident beam. The electrical resonance frequencies of samples $\mathrm{A}, \mathrm{B}$, and $\mathrm{C}$ were found at $3.63,3.81$, and $3.92 \mathrm{GHz}$, respectively.

Then, the transmission spectrum was acquired for the aperture [black line in Fig. 2(a)]. Since the aperture is too small in comparison to the wavelength of operation, we witnessed low transmission figures. ${ }^{3}$ However, a peak in the transmission spectrum around $3.7 \mathrm{GHz}$ was spotted once sample A was placed in the near field of the aperture [solid green line in Fig. 2(a)]. The experiment was repeated by replacing sample A with the CRR. CST Microwave Studio was used in order to realize the same configurations in the simulation environment. The results are illustrated in Fig. 2(b), which show the good agreement between the simulation and experimental outcomes. Figure 2 points out that the increase in the transmission figures was caused by effectively coupling the electric field component of the incoming field to the SRR structure. Sample A, which already had an electrical resonance around $3.63 \mathrm{GHz}$, triggered an enhanced transmission at $3.7 \mathrm{GHz}$. The absence of an electrical resonance for the CRR configuration prevented an extraordinary transmission within the same frequency band. Figure 3 exhibits the experimental and simulation based enhancement factors in linear scale when the same procedures were followed for samples $\mathrm{B}$ and $\mathrm{C}$. The transmission peaks occurred at 3.7 (sample A), 3.89 (sample B), and $4.01 \mathrm{GHz}$ (sample C), which coincide well with the electrical resonances of the

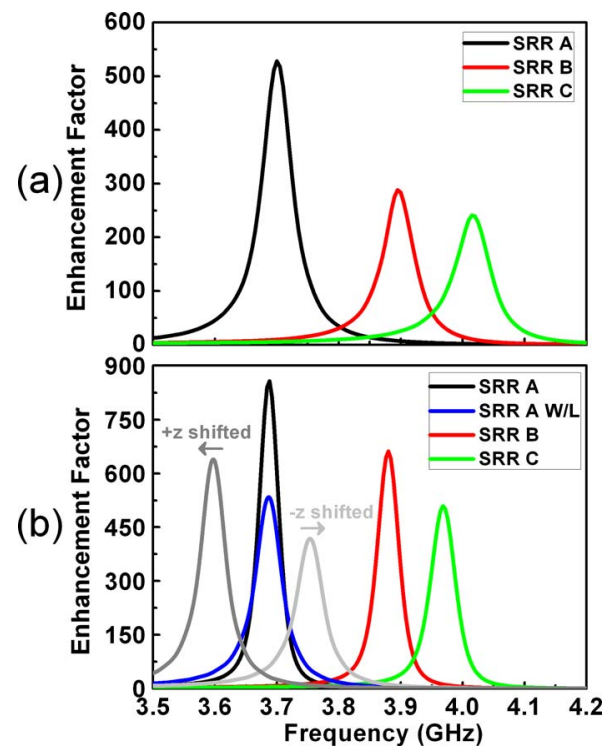

FIG. 3. (Color online) (a) Experimental and (b) numerical analysis of the enhancement figures for three different samples. Sample SRR A (solid black line), B (solid red line), and C (solid green line). Sample SRR A with the losses (solid blue line). Sample SRR A is shifted $-0.1 \mathrm{~mm}$ (solid light gray line) and $0.4 \mathrm{~mm}$ (solid gray line) in the $z$ direction with respect to origin of the aperture. The losses are also taken into account during the shifting procedure.

SRRs. Moreover, the measurements with the SRR-attached aperture yielded remarkable transmission improvement factors as high as 530, 288, and 232 for samples A, B, and C, respectively. Originally, the losses were not added to the calculations. The losses dropped the numerically computed enhancement factor to 540 from 856 for sample A [see Fig. 3 (b)] while broadening the enhancement peak when the metals are modeled with copper and the dielectrics with a loss tangent of $\delta=0.01$. The precise location of the SRR with respect to the aperture severely influences the transmission enhancement frequency. The enhancement turned out to be located at 3.59 and $3.75 \mathrm{GHz}$ when sample $\mathrm{A}$ is misaligned by 0.3 and $-0.1 \mathrm{~mm}$, respectively, from the origin of the aperture along the $z$ axis [solid gray and light gray lines in Fig. 3(b)].

The effect of the aperture radius on the enhancement factors was considered in Fig. 4. As the aperture radius ranged from 2.4 to $7 \mathrm{~mm}$, the simulation results showed that the transmission enhancement frequencies were also altered

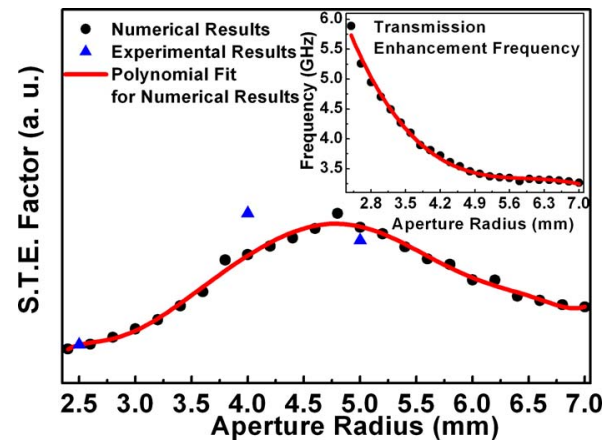

FIG. 4. (Color online) STE factor for various aperture radii, in the range of 2.4-7 mm. Numerical results (black dots), experimental results (blue triangles), and a polynomial fit curve for the numerical results (solid red curve). Inset: Calculated transmission enhancement frequencies corresponding to different aperture sizes. 


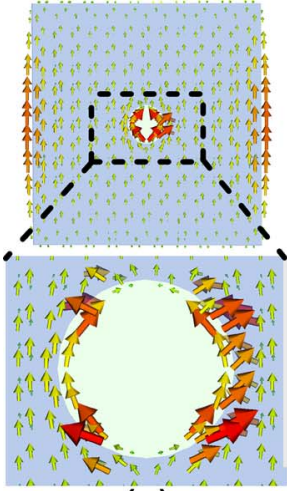

(a)

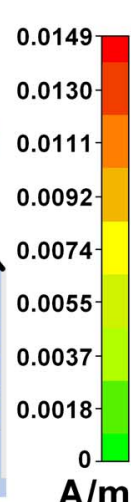

$\mathrm{A} / \mathrm{m}$

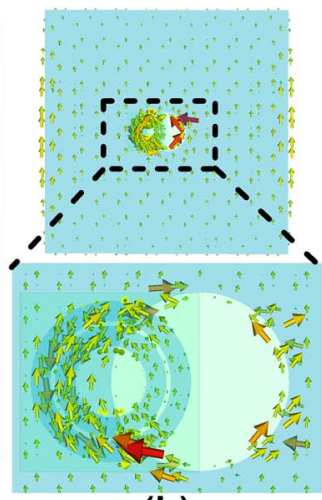

(b)

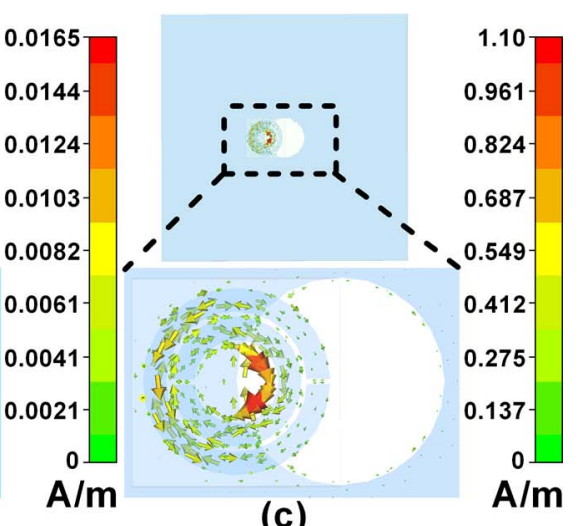

(c)
$\mathrm{A} / \mathrm{m}$

FIG. 5. (Color online) (a) The induced surface currents on the aperture, (b) aperture with CRR, and (c) aperture with sample SRR A. The region around the aperture is magnified for each case for clarification.

although the same SRR (sample A) and was employed throughout the entire analyses (see Fig. 4 inset). The EM response of the combined system, the aperture together with the SRR, had to be taken into account. The subwavelength transmission enhancement (STE) factor is defined as the multiplication of the transmission enhancement factors with the $(R / \lambda)$ values for different aperture radii ( $\lambda$ is the operational wavelength). The STE factor helps us to make a fair comparison to determine the optimal aperture geometry for the current investigation. Figure 4 reveals that the optimum aperture radius for this scheme is approximately $4.8 \mathrm{~mm}$, which corresponds to $1 / 18$ of the operational wavelength.

Inspection of the induced surface currents provides physical insight. Figure 5 shows the induced surface currents. The magnified images are especially taken around the aperture. According to Fig. 5(a), the surface currents on the metal block primarily remained parallel to the metal surface and attained the highest values at the discontinuities in the absence of the SRR. The weak contribution to the radiation came from the diffractions at the edges. The presence of the CRR did not cause any dramatic changes in Fig. 5(b). On the contrary, the surface currents turned out to be quantitatively amplified and focused around the aperture as soon as the CRR was substituted with the SRR at the enhancement frequency (see the color bars in Fig. 5). SRR, near the slit on the metal surface, concentrated the induced currents owing to its resonance behavior, acted like an antenna, and prepared the ground for a transmission enhancement through the opening. The metal block created image currents that nullified the induced surface currents on the SRR. Then, we can identify an effective antenna area that essentially involves the uncompensated induced surface currents on the SRR within the region overlapped by the aperture. The effective antenna area determines the enhancement frequency and the enhancement factors. This is the main reason why the sample SRR resonance frequencies did not perfectly coincide with the respective enhancement frequencies. We can further manipulate the effective antenna area by modifying the aperture radius. Smaller aperture radius values bring the enhancement to higher frequencies (see Fig. 4 inset). Misalignments gen- erate a similar outcome. The effective area is reduced by allowing the metal plate to cover a smaller portion of the SRR that in turn lessens the enhancement factors and increases the enhancement frequencies [see Fig. 3(b)].

In conclusion, we studied the extraordinary transmission by utilizing an electrically coupled SRR in the near field of a subwavelength aperture. The measurements and simulations demonstrated that a transmission enhancement figure of 530 could be achieved. This is a noteworthy accomplishment for an aperture with a radius of $1 / 20$ of the wavelength of operation when compared with previous experiments. ${ }^{4}$ Furthermore, we verified that the observed transmission enhancement effect is indeed due to the resonance behavior of the subwavelength resonant element by carrying out systematic experiments and numerical calculations.

This work is supported by the European Union under the projects EU-PHOME, and EU-ECONAM, and TUBITAK under Project Nos. 105A005, 106E198, and 107A004. One of the authors (E.O.) also acknowledges partial support from the Turkish Academy of Sciences.

${ }^{1}$ C. Genet and T. W. Ebbesen, Nature (London) 445, 39 (2007).

${ }^{2}$ T. Matsui, A. Agrawal, A. Nahata, and Z. V. Vardeny, Nature (London) 446, 517 (2007).

${ }^{3}$ H. A. Bethe, Phys. Rev. 66, 163 (1944).

${ }^{4}$ T. W. Ebbesen, H. J. Lezec, H. F. Ghaemi, T. Thio, and P. A. Wolff, Nature (London) 391, 667 (1998).

${ }^{5}$ L. Martin-Moreno, F. J. Garcia-Vidal, H. J. Lezec, K. M. Pellerin, T. Thio, J. B. Pendry, and T. W. Ebbesen, Phys. Rev. Lett. 86, 1114 (2001).

${ }^{6}$ W. L. Barnes, W. A. Murray, J. Dintinger, E. Devaux, and T. W. Ebbesen, Phys. Rev. Lett. 92, 107401 (2004).

${ }^{7}$ J. Barvo-Abad, A. Degiron, F. Przybilla, C. Genet, F. J. Garcia-Vidal, L. Martin-Moreno, and T. W. Ebbesen, Nat. Phys. 2, 120 (2006).

${ }^{8}$ A. Alu, F. Bilotti, N. Engetha, and L. Vegni, IEEE Trans. Antennas Propag. 54, 1632 (2006).

${ }^{9}$ N. Katsarakis, M. Kafesaki, I. Tsiapa, E. N. Economou, and C. M. Soukoulis, Photonics Nanostruct. Fundam. Appl. 5, 149 (2007).

${ }^{10}$ R. Marques, F. Mesa, J. Martel, and F. Medina, IEEE Trans. Antennas Propag. 51, 2572 (2003).

${ }^{11}$ K. Aydin, A. O. Cakmak, L. Sahin, Z. Li, F. Bilotti, L. Vegni, and E. Ozbay, Phys. Rev. Lett. 102, 013904 (2009). 